\title{
UN MODELO PARA EL ANÁLISIS SEMÁNTICO-DISCURSIVO DE LA CAUSALIDAD
}

\author{
ERnesto Wong García \\ Facultad de Lenguas Extranjeras, Universidad de La Habana, Cuba \\ Grupo de Estudios Semánticos e Ideográficos \\ ewong@flex.uh.cu
}

Recibido: 23/03/2015

Aceptado: 02/09/2015

\begin{abstract}
Resumen
La teoría dimensional del significado, desarrollada por el Dr. Leandro Caballero, tiene algunas limitaciones en lo que respecta a la codificación, a nivel de rasgos semánticos, de las relaciones de causalidad. Utilizando principalmente los métodos de análisis componencial y dimensional del significado, de modelación sobre trapecios semióticos y de análisis comparativo de corpus, este trabajo propone un modelo de escenarios causales lingüísticamente estructurados, basado en la dinámica de fuerzas, que amplía considerablemente la expresividad de los rasgos relacionales de la dimensión referencial. Concluye además que es posible tratar la causalidad como una supra-categoría semántico-nocional para su estudio tanto en el sistema de la lengua como en el discurso.

PALABRAS CLAVE: causalidad, teoría dimensional del significado, semántica, análisis del discurso.
\end{abstract}

\begin{abstract}
The dimensional theory of meaning, developed by Leandro Caballero, has some limitations when it comes to encoding causal relationships in semantic features. Using mainly the methods of componential and dimensional analysis of meaning, modelling on semiotic trapezoids and comparative corpus analysis, this paper proposes a model of linguistically structured causal scenarios, based on force-dynamics, which substantially increases feature expressiveness. It furthermore proposes to treat causality as an independent semantic and notional supra-category for its study both within the language system and in discourse.
\end{abstract}

KEYWORDS: causality, dimensional theory of meaning, semantics, discourse analysis.

\section{Introducción}

La teoría dimensional del significado, propuesta y desarrollada por el Dr. Leandro Caballero para el estudio de la modalidad como supra-categoría semántica, ha sido el centro del trabajo del Grupo de Estudios Semánticos e Ideográficos de nuestra Facultad de Lenguas Extranjeras (FLEX) durante los últimos veinte años. Estos estudios se han dedicado a las seis macro-categorías semánticas modales (MSM) propuestas por Caballero — valoración, lealtad, certidumbre, interés, afectividad y expresividad-, y han producido resultados notables, tanto teóricos (como la tesis doctoral de la Dra. Ana Ma. Galbán Pozo, dedicada a la verificación del carácter universal de las MSM) como prácticos (varios diccionarios ideográficos y semánticos (DIS) publicados y por publicar). 
La teoría tiene, sin embargo, algunas limitaciones. Una de ellas, a la que está dedicada la investigación, todavía en curso, cuyos resultados preliminares se presentan aquí, tiene que ver con el análisis semántico de las relaciones de causalidad. Éstas se codifican en la dimensión referencial, por medio de un grupo de rasgos llamados relacionales. En este trabajo muestro cómo estos rasgos no son suficientes para capturar el espectro de relaciones causales que se expresan en el lenguaje (sección 2). Apoyándome en la dinámica de fuerzas (sección 3), propongo un modelo de relaciones causales lingüísticamente estructurado (sección 4), y considero además otros tres aspectos que los hablantes parecen tomar en cuenta al enmarcar escenarios causales en el discurso, todo lo cual me lleva a señalar lo conveniente de tratar la causalidad como una supra-categoría semántico-nocional (sección 5). Finalmente, presento y comento un ejemplo de análisis de corpus utilizando el modelo propuesto (sección 6).

Los métodos principales de esta investigación han sido los de análisis componencial y dimensional del significado, el de modelación sobre trapecios semióticos y el de análisis comparativo de corpora.

\section{La teoría dimensional del significado}

Caballero concibió el significado lingüístico como una entidad compleja en la que se vinculan distintos tipos de contenidos: modales, ilocutivos, referenciales y formales, que se corresponden con cuatro dimensiones del significado: modal, ilocutiva, referencial y constructivo-gramatical, y que se caracterizan por conjuntos de rasgos semánticos específicos. Al abordar la problemática de la causalidad, me he centrado sobre todo en la dimensión referencial.

En la dimensión referencial «se enmarcan los rasgos de los referentes [...] considerados por los agentes del decir y que se caracterizan por una serie de grupos de rasgos, a saber: constitutivos, relacionales, de dominio y taxonomizadores» ${ }^{1}$. Aquí, me ocuparé sobre todo de los rasgos relacionales, que codifican «los diferentes tipos de relaciones que se establecen entre los objetos del mundo sobre la base de una perspectiva antropocéntrica $»^{2}$. Es fundamental insistir en el hecho de que no se trata de las relaciones objetivas en el mundo, sino de la percepción subjetiva que de ellas tienen los emisores del discurso.

Los rasgos relacionales que en la teoría dimensional del significado (TDS) se utilizan para codificar relaciones causales son los siguientes: rasgos de participación operacional, que incluyen causación (cambios de cualidades, cantidades, estados o comportamientos; hacer-ser), manipulación (influencia de un actante manipulador sobre otro para que este último realice una acción; hacer-hacer), concesión (vinculado a las nociones de permisión y perdón) y control (externo o interno, para evitar determinados estados de cosas); y el rasgo de experimentación de la operación, como contracara de las participaciones operacionales anteriores.

Esto presenta varios problemas. Primeramente, es difícil ver exactamente cuál es la diferencia entre la causación de un comportamiento y la manipulación. Segundo, como cada uno de estos rasgos admite una marca $[+]$ o $[-]$ (así como también $[ \pm]$ ), no está clara la diferencia

1 A. Ma. Galbán Pozo: «Aproximación al estudio de las macrocategorías semánticas modales...», p. 27.

2 Ibídem, pp. 27-28. 
entre, por ejemplo, [+CONTROL] y [-CONCESIÓN] o viceversa; la elección de uno u otro se deja al analista, según éste "sienta" la variante léxico-semántica (VLS) en cuestión. Tercero, el rasgo de experimentación de la operación intenta ser un rasgo altamente generalizador, pero a menudo se convierte en un passe partout que carece de la expresividad que se espera de un clasificador semántico. Incluso cuando se llegue a niveles de discretización más específicos (experimentación de la causación, de la manipulación, etc.), esto no hace sino reproducir los problemas que señalé para los rasgos de participación operacional. Y cuarto, el lenguaje expresa otras relaciones causales, a parte de las anteriores, que no hay manera de capturar por medio de estos rasgos. Tal es el caso de la noción de ayuda, en la que dos actantes actúan en la misma dirección, y que se diferencia de concesión por el carácter pasivo de la no interferencia en ésta, contrastado con el carácter activo de la cooperación en la primera.

Para intentar resolver estos y otros problemas, o al menos aumentar la capacidad expresiva de los rasgos relacionales en la TDS, me pareció pertinente considerar los estudios sobre la percepción de las relaciones causales que se han realizado en ciencia cognitiva, principalmente los referenciados en Jackendoff (2002) y Pinker (2008).

\section{La causalidad como dinámica de fuerzas}

Inicialmente propuesta y desarrollada por Talmy (1985), la dinámica de fuerzas se entiende como una categoría semántico-nocional que describe las interacciones de entidades haciendo referencia a la noción de fuerza. Aunque éste es un concepto de la física, la dinámica de fuerzas no se aplica solamente al dominio físico, sino que, como muchas otras nociones que subyacen las llamadas metáforas conceptuales ${ }^{3}$, se aplica también a otros como el psicológico o el epistémico.

La dinámica de fuerzas describe lo que he elegido llamar escenarios, en los cuales participan dos entidades: un agonista, en el cual se percibe una tendencia intrínseca al movimiento o al reposo; y un antagonista, que ejerce una fuerza sobre el agonista, generalmente contraria a la tendencia percibida de éste, pero que puede también reforzarla. En este caso, el antagonista actúa en concierto con el agonista. En los demás, si la fuerza ejercida es mayor, el agonista pasará del movimiento al reposo o viceversa; si no, seguirá haciendo lo que normalmente hace.

Talmy distingue también entre dos variantes de la causalidad: una variante estática, que nos presenta una "foto" de la situación, en la que agonista y antagonista siempre han existido juntos; y una variante dinámica, que nos presenta el momento en que el antagonista entra al escenario o sale, o el momento en que se resuelve la tensión entre los dos. Esta distinción está estrechamente relacionada con la categoría de aspecto y opone, en principio, las clases aspectuales Evento (aspecto télico) y Actividad o Estado (aspecto atélico) ${ }^{4}$. Aquí, las tomo como oposiciones paralelas, aunque no descarto que en etapas futuras de la investigación sea necesario un análisis más matizado.

3 Ver Cuenca y Hilferty (1999), principalmente el capítulo 4, "Metáfora y metonimia".

4 J. Moeschler: Introduction à la linguistique contemporaine, pp. 133-136. Una manera posible de entender la contrariedad télico :: atélico es como la diferencia entre dos maneras de conceptualizar una situación, según ésta tenga comienzo y/o final. La primera (télico) se aplica a eventos, que tienen un principio y/o un final, así como a los eventos mismos de comenzar o terminar. La segunda (atélico) se aplica a actividades y estados, que no tienen principio ni final. Otra manera, que no necesariamente niega ésta, y que es la que asumiré aquí, es la propuesta por Krifka (1998). Ver sección 5, sobre los rasgos de aspecto. 
Otros escenarios son también posibles: por ejemplo, uno en el que el antagonista tiende en la misma dirección que el agonista, esto es, actúan ambos en concierto, o uno en el que el antagonista no ejerce fuerza alguna sobre el agonista, aunque esté presente.

Nótese que la dinámica de fuerzas no se basa en ninguna teoría física o filosófica de la causalidad. Por el contrario, está construida sobre lo que podríamos llamar una teoría ingenua de la causalidad, que tiene mucho más en común con la teoría medieval del ímpetu que con la mecánica clásica newtoniana, y que nace de las intuiciones en que confiaron los primeros seres humanos en el proceso del conocimiento, y que son al fin y al cabo las que quedaron cristalizadas en la cognición, en el significado lingüístico y en las prácticas discursivas. Es éste modelo ingenuo de la causalidad el que debe intentar reflejar la teoría semántica.

\section{Un modelo de escenarios causales}

Combinando los posibles escenarios anteriores, y apoyándome en la técnica del trapecio semiótico — según la propuesta de Galbán Pozo $(2003)^{5}$ — , propongo el siguiente modelo de escenarios causales, representado en la figura 1.

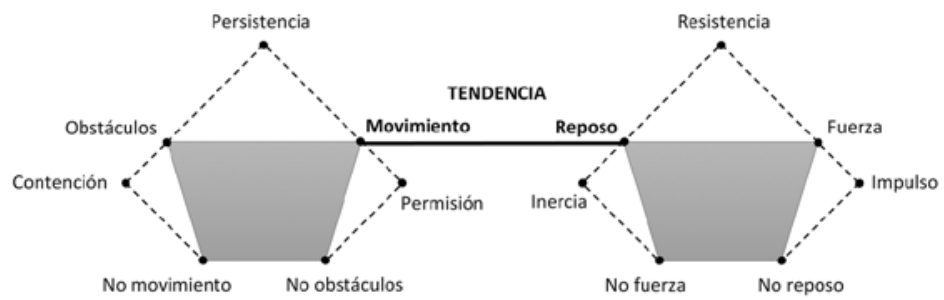

El modelo está construido sobre la oposición de los contrarios movimiento y reposo, que se refieren a la tendencia percibida del agonista. Cada uno de ellos genera a su vez su propio trapecio semiótico, al oponerse a otro contrario: movimiento :: obstáculos, y reposo :: fuerza, y al producir la oposición entre los correspondientes contradictorios no movimiento :: no obstáculos y no reposo :: no fuerza.

Ahora bien, es cierto que éstos no son contrarios en el sentido lexicológico de "antónimos". Sin embargo, es posible considerarlos contrarios semióticos en la medida en que se refieren a los comportamientos del agonista y del antagonista, respectivamente, en un escenario causal (EC), y en la medida en que, como se ve en la figura, sus conjunciones describen otros escenarios. Así, la conjunción de movimiento y obstáculos produce la noción de persistencia (movimiento a pesar de los obstáculos); la conjunción de fuerza y no reposo produce la noción de impulso (cambio hacia el movimiento); y, cruzando trapecios, la unión de movimiento y fuerza produce la noción de ayuda que resultaba imposible de representar en la dimensión referencial; entre otras.

5 Utilizar un trapecio, en lugar de la técnica tradicional del cuadrado semiótico creada por Greimas (1965) y adoptada por Caballero para el estudio de las MSM, permite, en caso de que lo doblásemos por la mitad de manera que contrarios y contradictorios quedaran sobre el mismo eje, reflejar el hecho de que la intensidad de la contradicción es menor que la intensidad de la contrariedad. En este caso, el uso del trapecio responde también a una necesidad lógica: no obstáculos no implica necesariamente movimiento, como no movimiento no implica necesariamente la presencia de obstáculos. Lo mismo se aplica al segundo trapecio. 
Aunque no estamos tratando con la mecánica clásica, inercia se refiere al concepto físico, tal como aparece enunciado en la Primera Ley de Newton, pero aplicado aquí solamente a un objeto cuya tendencia percibida es al reposo: Todo cuerpo persiste en su estado de reposo hasta que sea obligado a cambiar su estado por una fuerza ejercida sobre él. Se refiere entonces a un escenario en el que el antagonista "deja tranquilo" al agonista. Para los objetos cuya tendencia percibida es al movimiento, he preferido utilizar permisión.

Las conjunciones no movimiento :: no obstáculos y no reposo :: no fuerza no están marcadas porque producen, respectivamente, reposo y movimiento; volverlas a marcar sería redundante. Por otra parte, las conjunciones que no tienen opuestos - persistencia y resistencia - pueden marcarse en una matriz como $[+]$ o [-]. Además, como se demostrará en futuras etapas de esta investigación, todas estas conjunciones, contrarios y contradictorios pueden constituir la base de interacción con las MSM (ver además sección 6).

\section{La causalidad como supra-categoría semántico-nocional}

Incorporar las nociones de este modelo a la dimensión referencial como rasgos relacionales ya sería suficiente para aumentar de manera considerable su expresividad en cuanto a relaciones causales. Sin embargo, los hablantes parecen tomar en cuenta otros aspectos de estos escenarios a la hora de enmarcarlos en el discurso. Considérense los ejemplos siguientes:

(1) a. vencer a la resistencia b. resistir a toda costa

(2) el yugo imperial

(3) a. Estamos dispuestos a dialogar.

b. avenirse a razones

(4) a. ;No toleraremos otra derrota!

b. renunciar a sus principios

Los ejemplos en [1] nos presentan escenarios desde diferentes puntos de vista: [1a] desde el punto de vista del antagonista, y [1b] desde el del agonista. Además, [1a] nos presenta el momento en que se resuelve la tensión, mientras que [1b] nos presenta una imagen estática de la situación. El ejemplo en [2] nos presenta también una imagen estática, pero esta vez desde el punto de vista del antagonista. [3] nos muestra dos escenarios desde el punto de vista del agonista, pero [3a] es estático, mientras que [3b] es dinámico. Finalmente, [4a] y [4b] son ambos dinámicos, pero alternan entre los puntos de vista del antagonista y del agonista, respectivamente. Los ejemplos anteriores contienen además información sobre si el agonista logra o no realizar su tendencia intrínseca, y sobre si el antagonista logra o no alterar el comportamiento del agonista.

Tenemos entonces tres tipos de información que "delatan" la presencia de un hablante que enmarca los EC en el discurso desde su posición subjetiva: información sobre el actante categorial $^{6}$ focalizado (agonista o antagonista); sobre el aspecto del EC; y sobre el balance

6 Entiendo por actante categorial cada entidad "protagonista" de una supra-categoría semántico-nocional o de una macro-categoría semántica modal. En este caso, se refiere a las entidades participantes en un EC: agonista y antagonista. Para la MSM de valoración, serían el sujeto valorador y el objeto de la valoración; para interés, sería el sujeto interesado y el objeto de interés, etc. Los llamo actantes categoriales para distinguirlos de lo que 
final del EC. Toda esta información sugiere que estamos en presencia de algo mayor que un simple grupo de rasgos en la dimensión referencial.

La hipótesis que propongo considerar es la siguiente: La causalidad constituye una supra-categoría semántico-nocional (SSN).

Primeramente, es necesario explicar la terminología. ¿Por qué "semántico-nocional”? Porque estamos tratando con una región de la estructura conceptual que sirve para organizar tanto el contenido del pensamiento como el significado lingüístico. La problemática de la relación lenguaje-pensamiento-realidad se encuentra entonces en la base misma de esta investigación.

También debo explicitar qué entender por causalidad. De acuerdo con el modelo que propongo, y tratando de asegurarle la mayor aplicabilidad posible, entiendo por relación causal cualquier conceptualización lingüisticamente estructurada de una interacción entre dos entidades, de las cuales una exhiba un comportamiento en función de afectar la cualidad, cantidad / intensidad, comportamiento o estado de la otra (lo que en la TDS llamamos rasgos constitutivos).

La hipótesis anterior también tiene como consecuencia que las nociones que constituyen los nodos del modelo (Fig. 1) no sean atómicas, sino que tengan una estructura interna. Siguiendo a Talmy (1985), y luego a Jackendoff (2002), propongo estructurar los EC por medio de funciones de forma predicativo-argumental, que tomo como primitivas no descomponibles, y que divido en dos grupos: las funciones nucleares y las funciones complementarias.

Las funciones nucleares, como su nombre indica, estructuran el núcleo de la SSN de causalidad. Éstas son CAUS, LET y HELP.

- $\operatorname{CAUS}(\mathrm{x}, \mathrm{y})$ : El antagonista afecta al agonista. En la función, $\boldsymbol{x}$ es el antagonista, y $\boldsymbol{y}$ es una función complementaria.

- $\operatorname{LET}(\mathrm{x}, \mathrm{y})$ : El antagonista no se opone al agonista. Las variables toman los mismos valores que en $C A U S$.

- $\operatorname{HELP}(\mathrm{x}, \mathrm{y})$ : El antagonista refuerza la tendencia del agonista. Las variables toman los mismos valores que en CAUS y LET.

Las funciones complementarias ${ }^{7}$ son funciones básicas de la estructura conceptual que, en combinación con las funciones nucleares, permiten construir los EC. Las que propongo utilizar son BE, GO, STAY y CAMINO.

- $B E(\mathrm{x}, \mathrm{y})$ : (Categoría ontológica: Estado) Es la conceptualización de una configuración estática que se puede ubicar en un punto del tiempo o a lo largo de un período de tiempo.

- $G O(\mathrm{x}, \mathrm{y})$ : (Categoría ontológica: Evento) Es la conceptualización del evento en que $\boldsymbol{x}$ recorre $\boldsymbol{y}$. En la función, $\boldsymbol{x}$ toma los mismos valores que en $B E, \mathrm{y} \boldsymbol{y}$ es un Camino.

tradicionalmente se ha llamado actantes en las teorías de valencia. Estos actantes categoriales pueden realizarse como actantes (en el sentido tradicional), por ejemplo, como agentes, objetos, pacientes, experientivos, etc.

7 Esto no significa que las funciones en cuestión sean esencialmente complementarias, sólo que es ése su rol en este modelo. Es perfectamente posible, incluso probable, que funciones como GO, CAMINO y STAY sean funciones nucleares de una hipotética SSN de movimiento, por ejemplo, y que CAUS, LET y HELP tengan allí un rol complementario. El tratamiento de estas funciones ha sido tomado de Jackendoff (2002), pp. 394 en adelante. 
- $\operatorname{CAMINO}(\mathrm{w}, \mathrm{z})$ : (Categoría ontológica: Camino) En la versión de la función con la que estoy trabajando, $\boldsymbol{w}$ es el Origen y $\boldsymbol{z}$ es el Destino.

- $\operatorname{STAY}(\mathrm{x}, \mathrm{y})$ : (Categoría ontológica: Evento) Es la conceptualización de una configuración estática que se mantiene a lo largo de un período de tiempo. Las variables toman los mismos valores que en $B E$; de hecho, esta función es la "versión" eventiva de $B E$ (estativa).

A estos dos grupos de funciones es necesario también añadir operadores lógicos como NOT, que forman igualmente parte de la estructura conceptual, y que utilizamos aquí para modelar algunos EC.

$\mathrm{Al}$ relativizar, como hace Jackendoff, estas funciones complementarias por campo semántico (¿quizás "campo nocional” sería más apropiado?), se especifica el rango o tipo de valores que pueden tomar las variables (argumentos). Tomando como ejemplo la función $B E(\mathrm{x}, \mathrm{y})$, si la relativizamos al campo del espacio, $\boldsymbol{x}$ sería un Objeto, y $\boldsymbol{y}$ una Localización; en el campo de la posesión, $\boldsymbol{x}$ sería un Objeto, y $\boldsymbol{y}$ una persona que lo posee; en el campo de la atribución, $\boldsymbol{x}$ sería cualquier cosa, y $\boldsymbol{y}$ una Propiedad o Categoría a la que $\boldsymbol{x}$ pertenece.

Los principales EC del modelo quedarían entonces estructurados como sigue:

- Impulso: CAUS (Ant, GO (Ag, y))

- Contención: CAUS (Ant, STAY (Ag, y))

- Permisión: $L E T$ (Ant, GO (Ag, y))

- Inercia: NOT-CAUS (Ant, GO (Ag, y))

- Causación incoativa: CAUS (Ant, BE (Ag, y))

- Ayuda: HELP (Ant, GO (Ag, y)) - HELP (Ant, STAY (Ag, y))

- Persistencia: NOT-LET ( $\mathrm{Ag}_{\mathrm{i}}$, CAUS (Ant, STAY $\left.\left.\left(\mathrm{Ag}_{\mathrm{i}}, y\right)\right)\right)$

- Resistencia: NOT-LET (Ag,,$\left.C A U S\left(\mathrm{Ant}, G O\left(\mathrm{Ag}_{\mathrm{i}}, y\right)\right)\right)$

Nótese que he conservado el rasgo de causación que propone Caballero, pero aquí sólo se refiere a lo que he elegido llamar causación incoativa, esto es, de cambio de estado o, como lo llama Caballero, de hacer-ser. Los demás sustituyen y expanden el resto de las participaciones operacionales (manipulación, concesión, control, experimentación de la operación). Nótese también que, al contrario de los estudios tradicionales sobre la causalidad, en los que se habla de tipos de causalidad o de relaciones causales siempre en función del comportamiento del antagonista (causación, permisión, control, etc.), el modelo que propongo toma también en cuenta el comportamiento del agonista, y permite hablar de EC como persistencia o resistencia que, como lo veo yo, son también tipos de relaciones causales. Esto es precisamente lo que Caballero intentó hacer en la TDS con el rasgo relacional de experimentación de la operación.

También es conveniente contar con definiciones formales de los actantes categoriales de esta SSN. Podemos definir el agonista como una entidad participante en un escenario causal, que el emisor del discurso identifica como poseedora de una tendencia intrínseca al movimiento o al reposo. Por su parte, el antagonista sería una entidad participante en un escenario causal, que el emisor del discurso identifica como exhibidora de un comportamiento dirigido a afectar la cualidad, cantidad / intensidad, comportamiento o estado del agonista. 
La relación lógica entre ambas entidades es de presuposición. Agonista y antagonista sólo son tales en presencia uno del otro; es lógicamente imposible concebir un antagonista sin un agonista al que afectar, o un agonista sin un antagonista que lo afecte. Incluso en escenarios de inercia o de permisión, en los que el antagonista no afecta al agonista, el primero está presente y exhibe un comportamiento en función del segundo.

Más problemático es un enunciado como La revolución es imparable, en el que aparentemente se señala la ausencia de antagonista. Sin embargo, un análisis más detenido revela que, en la interpretación correcta (desde un punto de vista descriptivo, por supuesto, y no normativo), el antagonista se construye como una variable ligada por un cuantificador universal $(\forall x[\neg \diamond \text { parar }(x, r e v)])^{8}$, y cualquier entidad que satisfaga su valor participará como antagonista en un escenario de persistencia. El enunciado podría entonces parafrasearse como Nada de lo que existe es capaz de parar la revolución, o quizás de manera más precisa, Todo lo que existe es incapaz de parar la revolución. Vale señalar que, al parafrasear, hemos cambiado de actante categorial focalizado (del agonista al antagonista).

Según lo visto hasta ahora, tenemos cinco grupos de rasgos semánticos que son relevantes para el análisis de la SSN de causalidad, y que defino a continuación:

Rasgos de actante: Grupo de dos rasgos (agonista y antagonista) mutuamente excluyentes que reflejan el actante categorial que se focaliza en la VLS o en el enunciado para presentar el EC desde su punto de vista.

Rasgos de aspecto: Grupo de rasgos mutuamente excluyentes (aquí dos, télico y atélico, pero es posible concebir otras posibilidades como iterativo, por ejemplo) que reflejan la distribución en el tiempo del EC: atélico, si el EC se presenta como una situación, una configuración estática en la que el agonista y el antagonista siempre han existido juntos; o télico, si se presenta el momento en que el antagonista entra o sale del EC, o el momento en que se resuelve la tensión entre los dos.

Utilizo aquí los términos télico y atélico entendiéndolos según la propuesta, ya estándar, de Krifka (1998). Los EC atélicos serán aquellos para los que cualquier subparte de la eventualidad que describa el EC sea también una instancia del mismo EC (contribuir, intimidar, persistir, resistir). Por el contrario, los EC télicos sólo serán verdaderos de la eventualidad total que los describe, y no de ninguna de sus subpartes (ceder, claudicar, convencer, obligar). Como señalaba antes, esta concepción se corresponderá, en principio, con la oposición estático $: \because$ dinámico de Talmy (1985).

En dependencia del objeto del análisis (VLS aisladas o enunciados), debe cambiar el tipo de aspecto que se considera. Así, para las VLS aisladas estaremos hablando sobre todo de aspecto lexical, y de aspecto gramatical (composicional y morfológico) para los enunciados. De manera general, el tipo de aspecto a considerar dependerá de si el análisis semántico se circunscribe al sistema de la lengua (donde el aspecto está determinado por la naturaleza de la eventualidad) o al discurso (donde depende más de las elecciones del hablante).

8 Nótese el uso del operador para indicar la modalidad alética de imposibilidad, obtenida en este caso por la suma de los significados del sufijo - able y del prefijo -im. Esto es necesario para distinguir esta formulación de otra posible pero incorrecta, a saber, Nada de lo que existe está ahora parando la revolución. Estoy ignorando aquí la estructura del EC de contención que construye el verbo parar (pero ver anexo). 
Rasgos de balance final: Grupo de seis rasgos mutuamente excluyentes (éxito, fracaso, balance ambivalente, no éxito, no fracaso y balance ambiguo) que reflejan el balance final del EC: si el antagonista logra o no afectar al agonista, o si el agonista logra o no realizar su tendencia. Este grupo de rasgos se obtienen a partir de una modelación sobre trapecio semiótico, como muestra la figura 2.

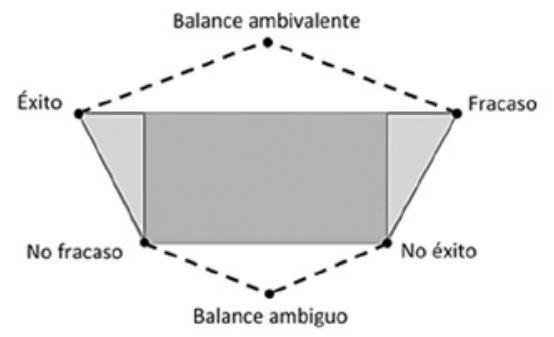

Es importante no axiologizar los contrarios y contradictorios, para no confundirnos con la MSM de valoración. Aunque los he llamado éxito y fracaso (o en el caso de cualquiera de los otros), estos nombres elegidos por conveniencia no deben interpretarse como portadores de una intención valorativa intrínseca: un éxito puede estar valorado como negativo ([2]), y un fracaso como positivo ([3a], [3b]).

No hay que confundir esta noción de balance final del EC con la de resultado del evento, utilizada en el análisis aspectual. Esta última se refiere a la relación que se establece entre un evento, el pre-estado que destruye, y el post-estado que crea9 ${ }^{9}$. La relación entre el evento y el post-estado es también causal, y se encuentra en la base de lo que aquí he llamado causación incoativa, o simplemente causación.

Rasgos de escenario: Grupo de doce rasgos (causación, movimiento, persistencia, obstáculos, contención, permisión, reposo, resistencia, fuerza, impulso, inercia y ayuda), no necesariamente excluyentes, que reflejan el EC en el que participa el actante focalizado, o el comportamiento de éste en un EC.

Rasgos de campo: Grupo de rasgos que relativizan los valores que pueden tomar los argumentos de las funciones que estructuran los EC. Constituyen un conjunto abierto, que podrá aumentarse según el nivel de discretización al que se aspire. Los principales serían espacio, tiempo, atribución, posesión, y psicológico. Este último es particularmente susceptible de subdividirse, ya que el valor que especifica para la variable $\boldsymbol{y}$ es uno de máxima generalidad, a saber, Estado Mental. Algunas subdivisiones posibles serían emocional, epistémico, volitivo, atencional, etc.

Estos cinco grupos de rasgos pueden sistematizarse en una matriz semántica, como en el análisis que se incluye en anexo, y que comento en la sección siguiente.

\section{Un ejemplo del modelo en uso}

A modo de ejemplo de la aplicación de este modelo, he querido incluir y comentar aquí un análisis de una pequeña muestra de 31 VLS aisladas que construyen EC, que deberá

9 J. Moeschler: op. cit., p. 135. 
servir para ilustrar las propuestas de este trabajo. Las definiciones lexicográficas con las que trabajé fueron tomadas del Diccionario de uso del español María Moliner (ver anexo 1).

Lo primero que hay que notar en este análisis es que muchas de las VLS consideradas son susceptibles de activar varios rasgos dentro de un mismo grupo. Tal es el caso, por ejemplo, de ceder $_{1}$, chantaje, convencer, exhortar, mover, prudente o testarudo. Esto se debe a que, en el sistema de la lengua, estas VLS están subespecificadas: poseen un rango de valores posibles, pero esa ambigüedad sólo se resuelve en el discurso. Cuando se realizan en enunciados completos, sí es posible determinar con exactitud los rasgos que se activan. Por ejemplo, convencer puede activar los rasgos de [+CONTENCIÓN] o [+IMPULSO] (entre otros); tomándola aislada, es imposible determinarlo. Sin embargo, si tomamos un enunciado como Lo convencí de que lo hiciera, se hace evidente que el EC que se construye es uno de impulso (mover a la acción, hacer-hacer).

Esta subespecificación también se refleja en los rasgos de campo. Para algunas VLS, la sección de rasgos de campo aparece sombreada. Esto significa que no es posible determinar cuál(es) de ellos se activan, pues la unidad aislada no lo especifica. Es así con contribuir, insuperable, irresistible, liberar, permitir o resistir. Otro caso es el de la VLS forzar ${ }_{2}$, que tampoco especifica un rasgo de campo, pero por razones distintas: en este caso, la VLS se refiere al comportamiento del antagonista (fuerza) en un EC, y no al EC completo. Como la variable $\boldsymbol{y}$ (cuyo valor debe relativizar el rasgo de campo) está en función del agonista, y al no considerarse aquí el agonista (aunque exista, recordemos que uno y otro se presuponen), tampoco es posible activar un rasgo de campo. En el caso de parar, el rasgo de campo podría ser [+ESPACIO], como en parar un tren, o [+TIEMPO], como en parar la huelga.

Las VLS ceder, y permitir ilustran el uso que hago de la noción de balance final y de los rasgos que le corresponden, así como también la diferencia entre ésta y la noción de resultado del evento, a la que hacía referencia arriba. En ceder ${ }_{l}$, lo que se focaliza es el fracaso del agonista al no realizar la tendencia intrínseca (reposo, entendido en el campo nocional psicológico o epistémico) que en él percibe el emisor de discurso. Independientemente del pre-estado que el evento de ceder, destruya y el post-estado que cree (que por demás no parecen evidentes), sigue siendo un hecho que el agonista de esta VLS no logra realizar su tendencia intrínseca. En el caso de permitir, se focaliza el fracaso, esta vez del antagonista, al no afectar la cualidad, cantidad / intensidad, comportamiento o estado del agonista. Aquí es también difícil ver cuáles serían el pre-estado y el post-estado del evento de permitir (¿agonista contenido y agonista libre?); sin embargo, el balance final del EC sí es determinable.

En otra línea, no muy alejada de ésta, las dos VLS anteriores contienen información sobre la intencionalidad (carácter deliberado) del comportamiento de los actantes categoriales: tanto ceder ${ }_{1}$ como permitir denotan acciones que, típicamente, se realizan de manera intencionada. Existen estudios ${ }^{10}$ sobre la construcción causativa en inglés que, tomando en cuenta la escala a la que se considera el evento, y los sub-eventos que se ignoran, sólo acepta verbos que se refieran a eventos causados de manera directa e intencionada. Lo que no queda claro es cómo esa intencionalidad se codifica en el significado del verbo y qué consecuencias tiene en la interpretación. Exactamente cuán relevante es esta información para el estudio de la SSN de causalidad en el sistema de la lengua (más allá de las restricciones de la construcción causativa) y en el discurso, es algo que habrá que determinar en futuras etapas de la investigación.

10 Por ejemplo, los de Wolff (2003), citados y comentados en Pinker (2008:107). 
También merecen comentario los pares de VLS prudente / cobarde y persistente / testarudo. En la matriz, estas VLS aparecen compuestas por los mismos rasgos (aunque testarudo puede, alternativamente, activar [+RESISTENCIA]), ya que denotan respectivamente los mismos comportamientos. La diferencia en la connotación (positiva para la primera VLS de cada par, negativa para la segunda) se resuelve al considerar la interacción de la SSN de causalidad y la MSM de valoración. Con las primeras VLS, el emisor de discurso hace una valoración apreciativa positiva del comportamiento de un agonista en un EC, mientras que con las segundas esta valoración es negativa ${ }^{11}$.

Otra interacción a considerar es la que se establece en la VLS convencer entre la SSN de causalidad y la MSM de certidumbre. Cuando esta VLS construye un EC de impulso, estructurado como CAUS (Ant, GO (AG, y)), y donde $\boldsymbol{y}$ es un Camino, las variables $\boldsymbol{w}$ y $\boldsymbol{z}$ (Origen y Destino) pueden corresponderse con las nociones contrarias que estructuran la MSM de certidumbre, a saber, los atributos modales indeciso y decidido para la perspectiva volitiva, e inseguro y seguro para la perspectiva cognoscitivo-discursiva ${ }^{12}$. El impulso puede entonces entenderse como "hacer ir de la indecisión a la decisión" (convencer a alguien de hacer algo) con un rasgo de campo [+PSICOLóGICo] (específicamente, [+volitivo]), o como "hacer ir de la inseguridad a la seguridad" (convencer a alguien de algo) con un rasgo de campo [+EPISTÉMICo]. La realización de la SSN de causalidad en las distintas MSM constituye el objeto de estudio principal de mi investigación actual.

Finalmente, la VLS liberar construye lo que he elegido llamar un escenario causal complejo (ECC), en el que un mismo actante categorial participa simultáneamente en dos EC. Como esta noción de ECC es también uno de los objetos de esta investigación en curso, el análisis que aparece en la matriz (dos filas, una para cada EC) es sólo tentativo.

\section{Conclusiones}

He señalado algunas limitaciones de la teoría dimensional del significado de L. Caballero, concretamente, limitaciones expresivas de los rasgos relacionales de la dimensión referencial, y he propuesto un modelo de escenarios causales, basado en la dinámica de fuerzas y modelado sobre trapecios semióticos. Además, he propuesto considerar la causalidad como una supra-categoría semántico-nocional, para la que he señalado tres aspectos que los hablantes toman en cuenta al enmarcar los escenarios causales en el discurso, y he incluido un ejemplo de análisis utilizando este modelo.

Éstos son sólo resultados preliminares de una investigación en curso que podría encontrar aplicaciones, por ejemplo, en el análisis del discurso político y/o periodístico — ¿qué nos dice la manera de construir y enmarcar un EC sobre la ideología del emisor?-, o en el análisis del discurso literario, en el que los EC pueden construir la estructura semántica narrativa.

11 Para un estudio más profundo de las MSM, y de valoración en específico, el lector interesado podrá consultar los trabajos referenciados del Dr. Leandro Caballero y de la Dra. Ana Ma. Galbán.

12 La perspectiva volitiva de la MSM de certidumbre refleja «el carácter de las actitudes (definidas vs indefinidas o dudosas) de los individuos en relación con los comportamientos a asumir» (Galbán Pozo, 2003:58). En la perspectiva cognoscitivo-discursiva «el hablante muestra en su discurso el grado de seguridad en relación con el conocimiento que tiene acerca de lo dicho» (Galbán Pozo, 2003:51). 


\section{Referencias bibliográficas}

Caballero, L. (2014): Semántica y diccionario. La Habana, Editorial de Ciencias Sociales.

- (2002): "Modalidades semánticas del lenguaje". En: Estudios lingüísticos cubanos (II) Homenaje a Leandro Caballero Díaz, Universitat de València, pp. 137-160.

Cuenca, M. J. y Hilferty, J. (1999): Introducción a la lingüística cognitiva, Barcelona, Editorial Ariel, S.A.

Galbán Pozo, A. Ma. (2003): “Aproximación al estudio de las macro-categorías semánticas modales (valoración, lealtad, certidumbre, interés, afectividad y expresividad) y su expresión a través de verbos de las lenguas española y alemana". Tesis de doctorado, La Habana.

Greimas, A. J. (1965): La sémantique structurale, París, Larousse.

Jackendoff, R. (2002): Foundations of Language, New York, Oxford University Press.

Krifka, M. (1998): “The Origins of Telicity". En: S. Rothstein (ed.): Events and grammar, Dordrecht, Kluwer Academic Press: http://amor.rz.hu-berlin.de/ h2816i3x/Publications/ TELICITY.pdf (06-06-2015).

María Moliner. Diccionario de uso del español. Edición electrónica, versión 3.0. Editorial Gredos, Madrid, 2008.

Moeschler, J. (2009): Introduction à la linguistique contemporaine, París, Armand Colin.

Pinker, S. (2008): The Stuff of Thought: Language as a Window into Human Nature, New York, Penguin Books.

Talmy, L. (1985): "Force-Dynamics in Language and Thought". Citado en Jackendoff (2002) y Pinker (2008).

Wolff, P. (2003): "Direct causation in the linguistic coding and individuation of causal events". En: Cognition, 88, pp. 1-48. Citado en Pinker (2008).

Wong, E. (2014): "Primer acercamiento al estudio de la macro-categoría semántica modal de interés en la lengua española". (Inédito) 


\section{Anexo 1: Muestra de variantes léxico-semánticas (VLS) aisladas \\ Fuente lexicográfica: María Moliner. Diccionario de uso del español.}

Boicotear: Privar a alguien, particularmente a una empresa industrial, de las relaciones o medios que necesita, para obligarle a ceder en cierta cosa.

Ceder ${ }_{1}$ : Cesar en una actitud de resistencia u oposición; por ejemplo, en una discusión o en una lucha.

Ceder $_{2}$ : Ponerse una cosa menos tensa o resistente. Romperse o soltarse algo sometido a una fuerza excesiva.

Chantaje: Procedimiento para conseguir algo que se pretende de una persona, amenazándola, particularmente con la difamación, si no accede a ello.

Claudicar: Ceder, rendirse o someterse; abandonar el esfuerzo o la resistencia en una empresa.

Coaccionar: Ejercer coacción sobre alguien para que haga cierta cosa.

Coacción: Cualquier procedimiento violento con que se obliga a alguien a hacer una cosa.

Cobarde: Se aplica a la persona que siente mucho miedo en los peligros o que no se atreve a exponerse a ellos, y, correspondientemente, a sus acciones, actitud, etc.

Coercer: Impedir a alguien que haga cierta cosa.

Coerción: Acción de reprimir por la fuerza.

Contribuir: Ser, junto con otras personas o cosas, causa de un suceso.

Convencer: Conseguir con razones que alguien se decida a hacer cierta cosa. Conseguir que alguien crea o piense cierta cosa.

Desistir: Abandonar cierta intención o proyecto, por necesidad, por convencerse de su imposibilidad o por cualquier consideración.

Exhortar: Inducir a alguien con palabras, razones o ruegos a que haga o deje de hacer una cosa.

Forzar $_{1}$ : Obligar a alguien, mediante la fuerza o cualquier clase de coacción, a que haga cosa contra su voluntad.

Forzar ${ }_{2}$ : Aplicar la fuerza a una cosa para hacer con ella cierta operación.

Forzar $_{3}$ : Esforzarse en que las cosas ocurran de manera distinta de como lo harían de manera natural, o más rápidamente.

Forzar $_{4}$ : Abrir algo, como una cerradura, una puerta o un cajón, por la fuerza.

Imparable: Que no se puede parar (ver infra).

Inamovible: No movible (ver mover).

Insuperable: No superable (ver superar).

Intimidar: Reducir a alguien a la acción por el temor.

Irresistible: Se aplica a lo que es imposible o inútil oponerle resistencia.

Liberar: Dejar libre algo o a alguien que estaba sujeto o preso.

Mover: Ser causa de que alguien haga cierta cosa, o contribuir a ello.

Obligar: Hacer que alguien realice cierta cosa usando para ello la fuerza o la autoridad o haciendo en cualquier forma que no tenga otro remedio que hacerla.

Parar: Hacer cesar un movimiento o una acción cualquiera. Detener una cosa oponiéndose a ella, antes de que llegue a su destino.

Permitir: No oponerse algo o alguien a que se realice u ocurra cierta cosa o a que alguien haga lo que se expresa.

Persistente: Que persiste.

Persistir: Seguir en cierta actitud u opinión.

Prudente: Aplicado a personas, a sus actos, palabras, se aplica al que obra con prudencia. Al que previene los peligros o los evita.

Prudencia: Moderación en el comportamiento para acomodarlo a lo que es sensato, discreto o exento de peligro.

Rendirse: Dejar de oponer resistencia en la guerra, en una lucha o en una discusión.

Resistir: No dejarse mover o influir por una fuerza u otra cosa.

Testarudo: Se aplica a la persona difícil de convencer, aun con razones convincentes, o a la que no se puede hacer desistir de cierta actitud. 


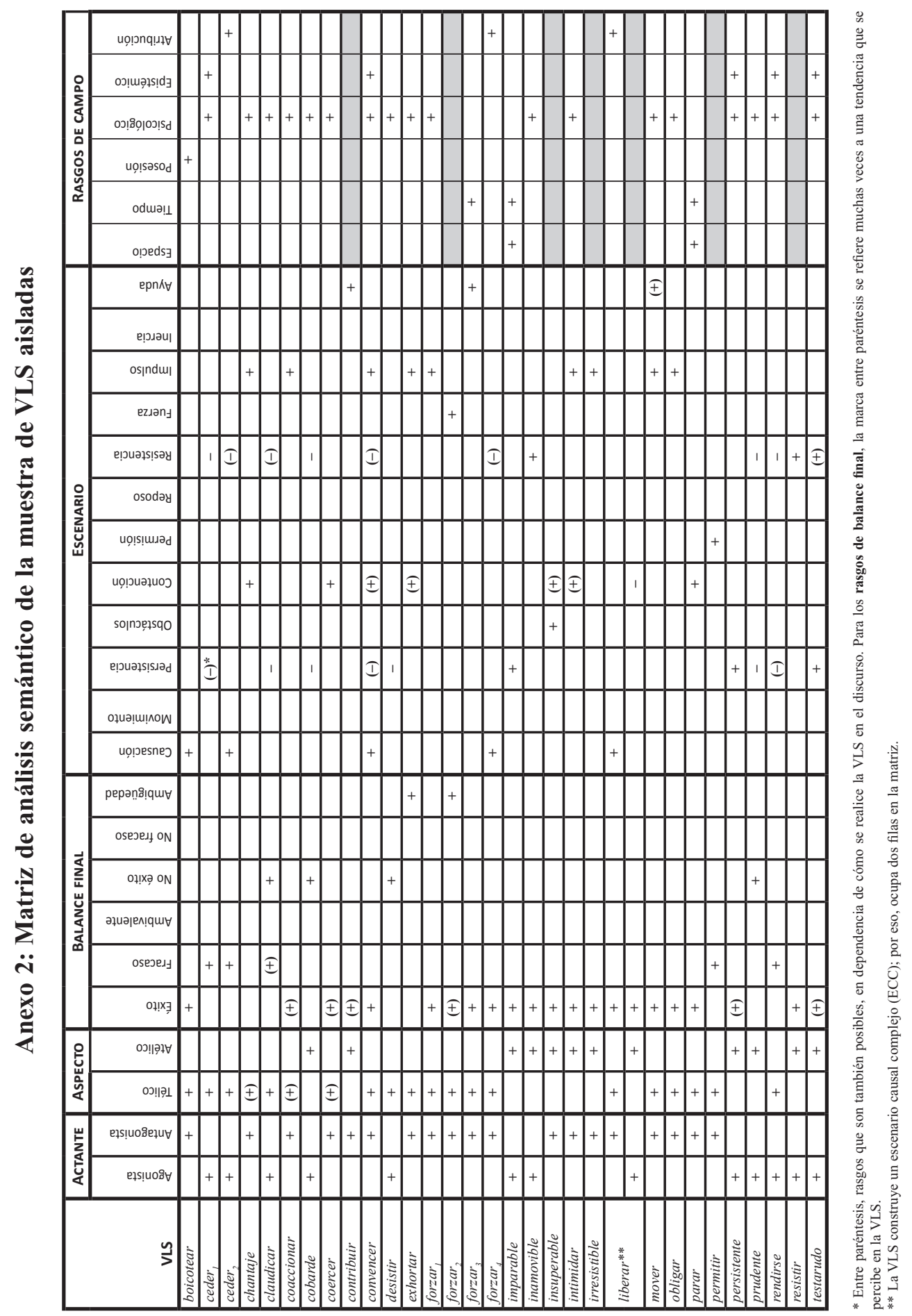

